\title{
Chronic care management of globesity: promoting healthier lifestyles in traditional and mHealth based settings
}

\author{
Gianluca Castelnuovo ${ }^{1,2 *}$, Giada Pietrabissa ${ }^{1,2}$, Gian Mauro Manzoni ${ }^{1,3}$, Stefania Corti ${ }^{1,4}$, \\ Martina Ceccarini ${ }^{1,4}$, Maria Borrello ${ }^{4}$, Emanuele M. Giusti ${ }^{2}$, Margherita Novelli ${ }^{1}$, \\ Roberto Cattivelli ${ }^{1}$, Nicole A. Middleton ${ }^{5}$, Susan G. Simpson ${ }^{5}$ and Enrico Molinari ${ }^{1,2}$ \\ 'Psychology Research Laboratory, Istituto Auxologico Italiano, Istituti di Ricovero e Cura a Carattere Scientifico, Ospedale \\ San Giuseppe, Verbania, Italy, ${ }^{2}$ Department of Psychology, Catholic University of Milan, Milan, Italy, ${ }^{3}$ Faculty of Psychology, \\ eCampus University, Milan, Italy, ${ }^{4}$ Department of Psychology, University of Bergamo, Bergamo, Italy, ${ }^{5}$ School of Psychology, \\ Social Work and Social Policy, University of South Australia, Adelaide, SA, Australia
}

\section{OPEN ACCESS}

Edited by:

Louise McHugh

National University of Ireland

Maynooth, Ireland

Reviewed by:

Michelle D. Keawphalouk,

Harvard-Massachusetts

Institute of Technology, USA

Omar C. G. Gelo,

Universita del Salento - Sigmuna

Freud University, Italy

*Correspondence:

Gianluca Castelnuovo gianluca.castelnuovo@auxologico.it

gianluca.castelnuovo@unicatt.it

Specialty section:

This article was submitted to

Psychology for Clinical Settings,

a section of the journal

Frontiers in Psychology

Received: 03 February 2015

Accepted: 25 September 2015

Published: 15 October 2015

Citation

Castelnuovo G, Pietrabissa G Manzoni GM, Corti S, Ceccarini M,

Borrello M, Giusti EM, Novelli M, Cattivelli R, Middleton NA, Simpson SG and Molinari E (2015) Chronic care management of globesity: promoting healthier lifestyles in traditional and $m$ Health based settings. Front. Psychol. 6:1557. doi: 10.3389/fpsyg.2015.01557
Obesity and being overweight could be real chronic conditions above all if there are other complications such as type 2 diabetes, cardiovascular diseases, hypertension, dyslipidemia, hypercholesterolemia, cancer, and various psychosocial and psychopathological disorders. Due to the multifactorial etiology of obesity, evidencebased interventions to improve weight loss, maintain a healthy weight, and reduce related comorbidities combine different treatment approaches: dietetic, nutritional, physical, behavioral, psychological, and, in some situations, pharmacological and surgical. There are significant limitations in this multidisciplinary chronic care management of obesity, most notably those regarding costs and long-term adherence and efficacy. Programs including eHealth platforms and new technologies could overcome limitations connected to the traditional in-patient chronic care management of obesity, thus providing promising opportunities in enhancing weight reduction and reducing complications in terms of long-term efficacy and effectiveness across clinical, organizational, and economic perspectives.

Keywords: obesity, type 2 diabetes, rehabilitation, mHealth, clinical psychology

\section{A MULTIDISCIPLINARY CHRONIC CARE MANAGEMENT OF GLOBESITY}

"Globesity" could be defined as a new global epidemic of obesity and being overweight with many associated complications and chronic conditions. Such conditions include not only type 2 diabetes, but also cardiovascular diseases, hypertension, dyslipidemia, hypercholesterolemia, cancer, and various psychosocial and psychopathological disorders (Wadden et al., 2002; Byrne et al., 2004; Flegal et al., 2005; Whitlock et al., 2009; Castelnuovo et al., 2014).

The etiology of obesity is universally recognized as multifactorial with a complex interaction between genetic, behavioral and environmental factors (Marcus and Wildes, 2009). Genetics plays an important role, but behavioral factors, such as dysfunctional eating habits and low levels physical activity, are among the main modifiable and proximal causes strictly connected to 
obesity and obesity-related complications (Dombrowski et al., 2012).

Due to the multifactorial etiology of obesity, evidencebased interventions to improve weight-loss, maintain a healthy weight, and reduce related comorbidities combine different treatment approaches: dietetic, nutritional, physical, behavioral, psychological, and, in some situations, pharmacological and surgical.

Clinical interventions, which typically focus on weight loss, reduction of obesity-related comorbidities, and change in dysfunctional behaviors, should be implemented in a multidisciplinary context with a clinical team composed of endocrinologists, nutritionists, dieticians, physiotherapists, psychiatrists, psychologists, and sometimes surgeons.

Also social and environmental barriers have to be considered in order to promote more effective strategies in weight loss programs (Rosas et al., 2015). For example policy interventions can potentially promote healthy behaviors (functional eating, physical activity) at the population level and not only in clinical settings (Barnidge et al., 2013). The socioeconomic situation of the patient has to be considered, in fact in developed countries the spread of obesity is more relevant than in lower socio-economic subgroups. Some specific reasons have been underlined by Mauro et al. (2008): "This has been attributed to the greater density of fast-food restaurants in lowincome neighbourhoods..., higher cost of healthy diets. .., safety concerns that prevent walking and other outdoor activities... and greater social acceptance of excess body weight. ... Affordability of membership in commercial weight-loss programs, gyms, obesity medications or surgery can likewise prove to be important obstacles. All of these factors can pose important barriers to weight management and interventions must specifically acknowledge and address these limitations" (p. 173). About child obesity, parents underline different obstacles to promote obesity prevention guidelines, such as child preferences, resistance to change habits, lack of knowledge and lack of a permanent monitoring attitude toward child behaviors, etc. (Sonneville et al., 2009).

Psychosocial and psychopathological determinants are key elements to consider in the successful long-term treatment of obesity due to the significant correlations between being overweight and self-esteem, quality of life, stress, life events, family and systemic scenarios, eating disorders, mood disorders, anxiety disorders, and personality disorders (Hudson et al., 2007; Pickering et al., 2007; Petry et al., 2008; Scott et al., 2008; Davin and Taylor, 2009; Manzoni et al., 2010).

For the in-patient management of obesity with or without type 2 diabetes, many psychological treatments are available, such as psychoeducational, cognitive-behavioral, interpersonal, systemicstrategic, and psychodynamic (Shaw et al., 2005).

Psychological therapies, typically focusing on dysfunctional behaviors, cognitive processes, unrealistic weight goals, and body image perceptions, could better help patients in achieving weight loss outcomes, both inside hospitals and clinical centers and during out-patient follow-up sessions (Wing, 2002; Swencionis and Rendell, 2012).
Some psychological skills should be implemented for functional chronic care management of obesity and its complications. Such skills include determining the client's ability to self-monitor (e.g., using diaries), assistance with stimulus control through restricting quantities of food, and behavioral modification strategies (e.g., chewing slowly, taking time to enjoy food, and increasing awareness of the pleasure associated with taste and food; Wing, 2002; Foster et al., 2005; Swencionis and Rendell, 2012). Specific psychological actions are also required in order to maintain goals that have initially been achieved, manage possible relapses, and learn strategies to cope with critical situations (Manzoni et al., 2010, 2011b; Dombrowski et al., 2011, 2012; Capodaglio et al., 2013).

\section{HIGH COSTS AND LONG-TERM PROBLEMS OF ADHERENCE IN TRADITIONAL IN-PATIENT CHRONIC CARE MANAGEMENT OF GLOBESITY}

Significant limitations in the multidisciplinary chronic care management of globesity concern costs and long-term adherence and efficacy. The obesity epidemic has been historically considered as a disease of high-income countries, but now is clearly characterizing also low- and middle-income countries with an increasing economic burden (Mitchell and Shaw, 2015). Although this scenario is critical, lifestyle interventions show promising results in terms of clinical evidence and costeffectiveness (Nugent, 2008; Boyers et al., 2015; Jerome et al., 2015; Whitfield et al., 2015).

Another limitation is the difficulty associated with maintaining long-term compliance and adherence in order to ensure clinical efficacy. "In fact, most overweight and obese individuals regain about one third of the weight lost with treatment within one year and they will typically come back to baseline in three to five years" (Pietrabissa et al., 2012, p. 317). Specifically in relation to the presence of diabetes complication, "Lifestyle intervention has been effective in several countries, but its success depends on uptake of intervention programmes and on compliance... An urgent priority is to identify ways to effectively engage people at risk of diabetes. Long-term sustainability is also a concern" (Zimmet et al., 2014, pp. 61-62).

Assessment of patients' motivation, compliance, and engagement is a key factor of treatment for obesity and its comorbidities (Waller et al., 2011; Barello et al., 2012). For example, the transtheoretical model of change (Prochaska and Diclemente, 1984; Astroth et al., 2002), which describes five motivational stages through which patients necessarily evolve while trying to change their dysfunctional behaviors, could be useful in explaining and predicting how and when individuals change their own unhealthy behaviors (Sarkin et al., 2001). Motivational Interviewing, defined as a "client-centred, directive method for enhancing intrinsic motivation to change by exploring and resolving ambivalence" (Wilson and Schlam, 2004), and Motivational Enhancement Therapy are other potential steps forward in comparison with the transtheoretical model (Miller and Johnson, 2001). 


\section{NEW TECHNOLOGIES FOR CHRONIC CARE MANAGEMENT OF GLOBESITY: THE mHealth PERSPECTIVE}

mHealth (also m-health, mHealth, or mobile health) could be defined as the practice of medicine and public health supported by mobile communication devices, such as mobile phones, tablet computers, and personal digital assistants (PDAs), for health services and information (Eysenbach, 2001, 2011; Riper et al., 2010; Cipresso et al., 2012; Whittaker, 2012; Fiordelli et al., 2013; Castelnuovo et al., 2014, 2015).

Programs with eHealth platforms and new technologies could overcome limitations connected to the traditional in-patient chronic care management of obesity with type 2 diabetes by providing promising opportunities for enhancing weight reduction and reducing complications in terms of long-term efficacy and effectiveness across clinical, organizational, and economic perspectives (Khaylis et al., 2010; Manzoni et al., 2011a; Rao et al., 2011). These technology-based strategies provided in out-patient settings are based on a collaborative approach derived from central planning and grounded in chronic care logic (Rao et al., 2011).

Particularly, considering the specific situation of obesity with type 2 diabetes, a detailed description of all possible mHealth applications is reported in Chomutare et al. (2011), where authors found more than 260 different diabetes applications (for Apple iPhone, Google Android, BlackBerry, and Nokia Symbian). All apps focused on the following features and measurements: self-monitoring, blood glucose, weight, physical activity, diet, insulin and medication, blood pressure, education, disease-related alerts and reminders, integration of social media functions, disease-related data export and communication, synchronization with personal health record (PHR) systems, and patient portals (Chomutare et al., 2011). Even if these apps are promising, new technologies have not yet demonstrated enough evidence in comparison with traditional approaches: for example computer-based self-management interventions for type 2 diabetes have only a limited beneficial effect on blood glucose control (a positive result is that this effect was more significant considering the mobile phone subgroup; Pal et al., 2013). Moreover these technologies have not yet produced significant impacts in other medical and psychological (cognitive, behavioral, emotional) variables (Pal et al., 2013).

Many clinical applications have been published about the utility of mobile phone devices in promoting healthy habits, weight loss attitudes, and reduction of comorbidities (Castelnuovo et al., 2010; Chomutare et al., 2011; Manzoni et al., 2011a; Rao et al., 2011; Simpson and Slowey, 2011; Burke et al., 2012; Cafazzo et al., 2012; Park and Kim, 2012; Pellegrini et al., 2012; Schiel et al., 2012; Bacigalupo et al., 2013; Fiordelli et al., 2013; Hebden et al., 2013; Martinez-Perez et al., 2013; Rodrigues et al., 2013; Schoffman et al., 2013; Sharifi et al., 2013; Shaw et al., 2013; Turner-McGrievy et al., 2013). One interesting project was the POWeR ("Positive Online Weight Reduction") Web-based weight management intervention that underlined the importance of supplementing new technologies based obesity treatment protocols with brief human support (Dennison et al., 2014). The mHealth approach has shown positive evidence not only in adult obesity (Tufano and Karras, 2005; Burke et al., 2012), but also in pediatric obesity (Jensen et al., 2012; TurnerMcGrievy et al., 2013). Applications have also been shown to increase participation, compliance, and engagement (Graffigna et al., 2013a,b).

Khaylis underlined five psychological components (Selfmonitoring, Counselor feedback and communication, Social support, Structured program, Individually-tailored program) necessary for functional mHealth-based chronic case management in order to facilitate weight loss and a reduction of comorbidities such as type 2 diabetes (Khaylis et al., 2010).

\section{BARRIERS TO THE EXPANSION OF mHealth CHRONIC CARE MANAGEMENT OF GLOBESITY}

Technical problems, skepticism, and reticence from patients, caregivers, nurses, and physicians could limit the growth of mHealth solutions (Gaggioli et al., 2005; Rees and Stone, 2005). Unfortunately, data collected about real costs of telemedicine are conflicting. Even so, mHealth could certainly reduce travel time, hospital admissions, and indirect costs for service users and their families and social networks.

Mohammadzadeh and Safdari (2014) summarized the main barriers and challenges in the development of mHealth scenarios in chronic care management of different pathologies:

- Organizational and technological barriers

- User attitudes

- Technology acceptance

- Threats to confidentiality and privacy

- Legal, ethical, and administrative barriers

- Costs of system implementation

- Costs of system maintenance

- Lack of sufficient investment

- Poor design and implementation

- Lack of system interoperability with electronic health records and other IT tools

- Decrease in face-to-face communication between doctor and patient

- Poor functioning of system that leads to medical errors and negative effects on care outcomes, patients and personnel

- Mistakes in documentation; misrepresentation

- Data manipulation and violation of patients' legal rights

- Reliability, sustainability of connections, sudden interruptions of telecommunication networks

- Scalability in terms of data rate and power and energy consumption

- Antenna design, quality of service (QoS), energy efficiency

- Weight of wearable devices

- Training user to use wearable system

- Wearable system market penetration 


\section{FUTURE DIRECTIONS IN CLINICAL PSYCHOLOGY AND MEDICINE FOR CHRONIC CARE MANAGEMENT OF GLOBESITY}

Turner-McGrievy noted that potential benefits of mobile monitoring methods for behavioral weight loss protocols appear clear: "future studies should examine ways to predict which selfmonitoring method works best for an individual to increase adherence" (Turner-McGrievy et al., 2013, p. 513). There is a critical need for scientific research to evaluate the specific outcomes of collaborative approaches for weight management that utilize the Internet and mobile-based tools.

The mHealth approach could help clinicians by motivating patients in remote settings to develop healthier lifestyles (Pietrabissa et al., 2012), to accept more intrusive medical treatments, and to cope with chronic conditions by reducing complications (such as type 2 diabetes, hypertension, and cardiovascular disease; Nguyen and Lau, 2012).

Further studies are required to evaluate the feasibility of the chronic care model as a perspective in obesity interventions (Rao et al., 2011). It is imperative that clinical psychology and medicine develop new diagnostic, monitoring and treatment protocols usable in both traditional and innovative technology-based settings, incorporating recent scientific and clinical evidence (Pietrabissa et al., 2012) and respectful communication (Kahn, 2008; Macagno and Walton, 2010; Bigi, 2011; Castelnuovo, 2013) even in an mHealth scenario.

One promising future direction could be treating obesity, considered as a chronic multifactorial disease, using a steppedcare approach (Kushner, 2014; Castelnuovo et al., 2015):

\section{REFERENCES}

Astroth, D. B., Cross-Poline, G. N., Stach, D. J., Tilliss, T. S., and Annan, S. D. (2002). The transtheoretical model: an approach to behavioral change. J. Dent. Hyg. 76, 286-295.

Bacigalupo, R., Cudd, P., Littlewood, C., Bissell, P., Hawley, M. S., and Buckley Woods, H. (2013). Interventions employing mobile technology for overweight and obesity: an early systematic review of randomized controlled trials. Obes. Rev. 14, 279-291. doi: 10.1111/obr.12006

Barello, S., Graffigna, G., and Vegni, E. (2012). Patient engagement as an emerging challenge for healthcare services: mapping the literature. Nurs. Res. Pract. 2012, 905934. doi: 10.1155/2012/905934

Barnidge, E. K., Radvanyi, C., Duggan, K., Motton, F., Wiggs, I., Baker, E. A., et al. (2013). Understanding and addressing barriers to implementation of environmental and policy interventions to support physical activity and healthy eating in rural communities. J. Rural Health 29, 97-105. doi: 10.1111/j.17480361.2012.00431.x

Bigi, S. (2011). The persuasive role of ethos in doctor-patient interactions. Commun. Med. 8, 67-76. doi: 10.1558/cam.v8i1.67

Boyers, D., Avenell, A., Stewart, F., Robertson, C., Archibald, D., Douglas, F., et al. (2015). A systematic review of the cost-effectiveness of nonsurgical obesity interventions in men. Obes. Res. Clin. Pract. 9, 310-327. doi: 10.1016/j.orcp.2015.03.001

Burke, L. E., Styn, M. A., Sereika, S. M., Conroy, M. B., Ye, L., Glanz, K., et al. (2012). Using mHealth technology to enhance self-monitoring for weight loss: a randomized trial. Am. J. Prev. Med. 43, 20-26. doi: 10.1016/j.amepre.2012.03.016

Byrne, S. M., Cooper, Z., and Fairburn, C. G. (2004). Psychological predictors of weight regain in obesity. Behav. Res. Ther. 42, 1341-1356. doi: 10.1016/j.brat.2003.09.004
- The lower level of treatment could be simply a mHealth or traditional based lifestyle psychoeducational and nutritional approach to weight management. This step could be also considered as a prevention phase at the population level.

- The following step, useful in moderate conditions, could be represented by the inclusion in health professionalsdriven multidisciplinary protocols tailored for each patient. The mHealth approach could be useful for providing many parts of the clinical program reducing costs and limiting hospitalizations.

- Another step of care, useful in severe conditions, could be the inpatient approach with the inclusion of drug therapies and other multidisciplinary tretaments if necessary. The mHealth contribution could be useful for monitoring and motivating patients after the inpatient phase and for reducing costs above all in the follow-up steps.

- Another step, in more severe conditions, needs the solution of bariatric surgery and with this option the mHealth approach could be useful for monitoring eating attitudes, motivating patients in changing dysfunctional behaviors and for reducing costs above all in the follow-up steps.

In the chronic care management of globesity mHealth solutions cannot substitute traditional approaches, but they can supplement some steps in obesity prevention and weight loss management, above all in the follow-up phase where the mobile technology can ensure a continuity of care saving costs and avoiding long term lack of connections between patientscitizens and the health care team.

Cafazzo, J. A., Casselman, M., Hamming, N., Katzman, D. K., and Palmert, M. R. (2012). Design of an mHealth app for the self-management of adolescent type 1 diabetes: a pilot study. J. Med. Internet Res. 14, e70. doi: 10.2196/jmir.2058

Capodaglio, P., Lafortuna, C., Petroni, M. L., Salvadori, A., Gondoni, L., Castelnuovo, G., et al. (2013). Rationale for hospital-based rehabilitation in obesity with comorbidities. Eur. J. Phys. Rehabil. Med. 49, 399-417.

Castelnuovo, G. (2013). 5 years after the Kahn's etiquette-based medicine: a brief checklist proposal for a functional second meeting with the patient. Front. Psychol. 4:723. doi: 10.3389/fpsyg.2013.00723

Castelnuovo, G., Manzoni, G. M., Cuzziol, P., Cesa, G. L., Tuzzi, C., Villa, V., et al. (2010). TECNOB: study design of a randomized controlled trial of a multidisciplinary telecare intervention for obese patients with type-2 diabetes. BMC Public Health 10:204. doi: 10.1186/1471-2458-10-204

Castelnuovo, G., Manzoni, G. M., Pietrabissa, G., Corti, S., Giusti, E. M., Molinari, E., et al. (2014). Obesity and outpatient rehabilitation using mobile technologies: the potential mHealth approach. Front. Psychol. 5:559. doi: 10.3389/fpsyg.2014.00559

Castelnuovo, G., Zoppis, I., Santoro, E., Ceccarini, M., Pietrabissa, G., Manzoni, G. M., et al. (2015). Managing chronic pathologies with a stepped mHealthbased approach in clinical psychology and medicine. Front. Psychol. 6:407. doi: 10.3389/fpsyg.2015.00407

Chomutare, T., Fernandez-Luque, L., Arsand, E., and Hartvigsen, G. (2011) Features of mobile diabetes applications: review of the literature and analysis of current applications compared against evidence-based guidelines. J. Med. Internet Res. 13, e65. doi: 10.2196/jmir.1874

Cipresso, P., Serino, S., Villani, D., Repetto, C., Selitti, L., Albani, G., et al. (2012). Is your phone so smart to affect your states? An exploratory study based on psychophysiological measures. Neurocomputing 84, 23-30. doi: 10.1016/j.neucom.2011.12.027 
Davin, S. A., and Taylor, N. M. (2009). Comprehensive review of obesity and psychological considerations for treatment. Psychol. Health Med. 14, 716-725. doi: 10.1080/13548500903431501

Dennison, L., Morrison, L., Lloyd, S., Phillips, D., Stuart, B., Williams, S., et al. (2014). Does brief telephone support improve engagement with a web-based weight management intervention? Randomized controlled trial. J. Med. Internet Res. 16, e95. doi: 10.2196/jmir.3199

Dombrowski, S. U., Avenell, A., and Sniehott, F. F. (2011). Behavioural interventions for obese adults with additional risk factors for morbidity: systematic review of effects on behaviour, weight and disease risk factors. Obes. Facts 3, 377-396. doi: $10.1159 / 000323076$

Dombrowski, S. U., Sniehotta, F. F., Avenell, A., Johnston, M., Maclennan, G., and Araújo-Soares, V. (2012). Identifying active ingredients in complex behavioural interventions for obese adults with obesity-related co-morbidities or additional risk factors for co-morbidities: a systematic review. Health Psychol. Rev. 6, 7-32. doi: 10.1080/17437199.2010.513298

Eysenbach, G. (2001). What is e-health? J. Med. Internet Res. 3, E20. doi: 10.2196/jmir.3.2.e20

Eysenbach, G. (2011). Can tweets predict citations? Metrics of social impact based on Twitter and correlation with traditional metrics of scientific impact. J. Med. Internet Res. 13, e123. doi: 10.2196/jmir.2012

Fiordelli, M., Diviani, N., and Schulz, P. J. (2013). Mapping mHealth research: a decade of evolution. J. Med. Internet Res. 15, e95. doi: 10.2196/jmir.2430

Flegal, K. M., Graubard, B. I., Williamson, D. F., and Gail, M. H. (2005). Excess deaths associated with underweight, overweight, and obesity. JAMA 293, 1861-1867. doi: 10.1001/jama.293.15.1861

Foster, G. D., Makris, A. P., and Bailer, B. A. (2005). Behavioral treatment of obesity. Am. J. Clin. Nutr. 82, 230S-235S.

Gaggioli, A., Di Carlo, S., Mantovani, F., Castelnuovo, G., and Riva, G. (2005). A telemedicine survey among Milan doctors. J. Telemed. Telecare 11, 29-34. doi: $10.1258 / 1357633053430476$

Graffigna, G., Barello, S., and Riva, G. (2013a). Technologies for patient engagement. Health Aff. (Millwood) 32, 1172. doi: 10.1377/hlthaff.2013.0279

Graffigna, G., Barello, S., Wiederhold, B. K., Bosio, A. C., and Riva, G. (2013b). Positive technology as a driver for health engagement. Stud. Health Technol. Inform. 191, 9-17.

Hebden, L., Balestracci, K., Mcgeechan, K., Denney-Wilson, E., Harris, M., Bauman, A., et al. (2013). 'TXT2BFiT' a mobile phone-based healthy lifestyle program for preventing unhealthy weight gain in young adults: study protocol for a randomized controlled trial. Trials 14, 75. doi: 10.1186/1745-621514-75

Hudson, J. I., Hiripi, E., Pope, H. G. Jr., and Kessler, R. C. (2007). The prevalence and correlates of eating disorders in the National Comorbidity Survey Replication. Biol. Psychiatry 61, 348-358. doi: 10.1016/j.biopsych.2006.03.040

Jensen, C. D., Aylward, B. S., and Steele, R. G. (2012). Predictors of attendance in a practical clinical trial of two pediatric weight management interventions. Obesity (Silver Spring) 20, 2250-2256. doi: 10.1038/oby.2012.96

Jerome, G. J., Alavi, R., Daumit, G. L., Wang, N. Y., Durkin, N., Yeh, H. C., et al. (2015). Willingness to pay for continued delivery of a lifestyle-based weight loss program: the Hopkins POWER trial. Obesity (Silver Spring) 23, 282-285. doi: 10.1002/oby.20981

Kahn, M. W. (2008). Etiquette-based medicine. N. Engl. J. Med. 358, 1988-1989. doi: 10.1056/NEJMp0801863

Khaylis, A., Yiaslas, T., Bergstrom, J., and Gore-Felton, C. (2010). A review of efficacious technology-based weight-loss interventions: five key components. Telemed. J. E Health 16, 931-938. doi: 10.1089/tmj.2010.0065

Kushner, R. F. (2014). Weight loss strategies for treatment of obesity. Prog. Cardiovasc. Dis. 56, 465-472. doi: 10.1016/j.pcad.2013.09.005

Macagno, F., and Walton, D. (2010). What we hide in words: emotive words and persuasive definitions. J. Pragmat. 42, 1997-2013. doi: 10.1016/j.pragma.2009.12.003

Manzoni, G. M., Cribbie, R. A., Villa, V., Arpin-Cribbie, C. A., Gondoni, L., and Castelnuovo, G. (2010). Psychological well-being in obese inpatients with ischemic heart disease at entry and at discharge from a four-week cardiac rehabilitation program. Front. Psychol. 1:38. doi: 10.3389/fpsyg.2010.00038

Manzoni, G. M., Pagnini, F., Corti, S., Molinari, E., and Castelnuovo, G. (2011a). Internet-based behavioral interventions for obesity: an updated systematic review. Clin. Pract. Epidemiol. Ment. Health 7, 19-28. doi: 10.2174/1745017901107010019
Manzoni, G. M., Villa, V., Compare, A., Castelnuovo, G., Nibbio, F., Titon, A. M., et al. (2011b). Short-term effects of a multi-disciplinary cardiac rehabilitation programme on psychological well-being, exercise capacity and weight in a sample of obese in-patients with coronary heart disease: a practice-level study. Psychol. Health Med. 16, 178-189. doi: 10.1080/13548506.2010.542167

Marcus, M. D., and Wildes, J. E. (2009). Obesity: is it a mental disorder? Int. J. Eat. Disord. 42, 739-753. doi: 10.1002/eat.20725

Martinez-Perez, B., De La Torre-Diez, I., and Lopez-Coronado, M. (2013). Mobile health applications for the most prevalent conditions by the World Health Organization: review and analysis. J. Med. Internet Res. 15, e120. doi: 10.2196/jmir.2600

Mauro, M., Taylor, V., Wharton, S., and Sharma, A. M. (2008). Barriers to obesity treatment. Eur. J. Intern. Med. 19, 173-180. doi: 10.1016/j.ejim.2007.09.011

Miller, C. E., and Johnson, J. L. (2001). Motivational interviewing. Can. Nurse 97, $32-33$.

Mitchell, S., and Shaw, D. (2015). The worldwide epidemic of female obesity. Best Pract. Res. Clin. Obstet. Gynaecol. 29, 289-299. doi: 10.1016/j.bpobgyn.2014.10.002

Mohammadzadeh, N., and Safdari, R. (2014). Patient monitoring in mobile health: opportunities and challenges. Med. Arch. 68, 57-60.

Nguyen, T., and Lau, D. C. (2012). The obesity epidemic and its impact on hypertension. Can. J. Cardiol. 28, 326-333. doi: 10.1016/j.cjca.2012.01.001

Nugent, R. (2008). Chronic diseases in developing countries: health and economic burdens. Ann. N. Y. Acad. Sci. 1136, 70-79. doi: 10.1196/annals.1425.027

Pal, K., Eastwood, S. V., Michie, S., Farmer, A. J., Barnard, M. L., Peacock, R., et al. (2013). Computer-based diabetes self-management interventions for adults with type 2 diabetes mellitus. Cochrane Database Syst. Rev. 3, CD008776. doi: 10.1002/14651858.CD008776.pub2

Park, M. J., and Kim, H. S. (2012). Evaluation of mobile phone and Internet intervention on waist circumference and blood pressure in post-menopausal women with abdominal obesity. Int. J. Med. Inform. 81, 388-394. doi: 10.1016/j.ijmedinf.2011.12.011

Pellegrini, C. A., Duncan, J. M., Moller, A. C., Buscemi, J., Sularz, A., Demott, A., et al. (2012). A smartphone-supported weight loss program: design of the ENGAGED randomized controlled trial. BMC Public Health 12:1041. doi: 10.1186/1471-2458-12-1041

Petry, N. M., Barry, D., Pietrzak, R. H., and Wagner, J. A. (2008). Overweight and obesity are associated with psychiatric disorders: results from the National Epidemiologic Survey on Alcohol and Related Conditions. Psychosom. Med. 70, 288-297. doi: 10.1097/PSY.0b013e3181651651

Pickering, R. P., Grant, B. F., Chou, S. P., and Compton, W. M. (2007). Are overweight, obesity, and extreme obesity associated with psychopathology? Results from the national epidemiologic survey on alcohol and related conditions. J. Clin. Psychiatry 68, 998-1009. doi: 10.4088/JCP.v68n0704

Pietrabissa, G., Manzoni, G., Corti, S., Vegliante, N., Molinari, E., and Castelnuovo, G. (2012). Addressing motivation in globesity treatment: a new challenge for clinical psychology. Front. Psychol. 3:317. doi: 10.3389/fpsyg.2012.00317

Prochaska, J. O., and Diclemente, C. C. (1984). The Transtheoretical Approach: Crossing Traditional Boundaries of Change. Homewood, IL: Dorsey Press.

Rao, G., Burke, L. E., Spring, B. J., Ewing, L. J., Turk, M., Lichtenstein, A. H., et al. (2011). New and emerging weight management strategies for busy ambulatory settings: a scientific statement from the American Heart Association endorsed by the Society of Behavioral Medicine. Circulation 124, 1182-1203. doi: 10.1161/CIR.0b013e31822b9543

Rees, C. S., and Stone, S. (2005). Therapeutic alliance in face-to-face versus videoconference psychotherapy. Prof. Psychol. Res. Pr. 6, 5. doi: 10.1037/07357028.36.6.649

Riper, H., Andersson, G., Christensen, H., Cuijpers, P., Lange, A., and Eysenbach, G. (2010). Theme issue on e-mental health: a growing field in internet research. J. Med. Internet Res. 12, e74. doi: 10.2196/jmir.1713

Rodrigues, J. J., Lopes, I. M., Silva, B. M., and Torre Ide, L. (2013). A new mobile ubiquitous computing application to control obesity: SapoFit. Inform. Health Soc. Care 38, 37-53. doi: 10.3109/17538157.2012.674586

Rosas, L. G., Thiyagarajan, S., Goldstein, B. A., Drieling, R. L., Romero, P. P., Ma, J., et al. (2015). The effectiveness of two community-based weight loss strategies among obese, low-income US latinos. J. Acad. Nutr. Diet. 115, 537-550.e2. doi: 10.1016/j.jand.2014.10.020

Sarkin, J. A., Johnson, S. S., Prochaska, J. O., and Prochaska, J. M. (2001). Applying the transtheoretical model to regular moderate exercise in an overweight 
population: validation of a stages of change measure. Prev. Med. 33, 462-469. doi: 10.1006/pmed.2001.0916

Schiel, R., Kaps, A., and Bieber, G. (2012). Electronic health technology for the assessment of physical activity and eating habits in children and adolescents with overweight and obesity IDA. Appetite 58, 432-437. doi: 10.1016/j.appet.2011.11.021

Schoffman, D. E., Turner-Mcgrievy, G., Jones, S. J., and Wilcox, S. (2013). Mobile apps for pediatric obesity prevention and treatment, healthy eating, and physical activity promotion: just fun and games? Transl. Behav. Med. 3, 320-325. doi: 10.1007/s13142-013-0206-3

Scott, K. M., Bruffaerts, R., Simon, G. E., Alonso, J., Angermeyer, M., De Girolamo, G., et al. (2008). Obesity and mental disorders in the general population: results from the world mental health surveys. Int. J. Obes. (Lond.) 32, 192-200. doi: 10.1038/sj.ijo.0803701

Sharifi, M., Dryden, E. M., Horan, C. M., Price, S., Marshall, R., Hacker, K., et al. (2013). Leveraging text messaging and mobile technology to support pediatric obesity-related behavior change: a qualitative study using parent focus groups and interviews. J. Med. Internet Res. 15, e272. doi: 10.2196/ jmir. 2780

Shaw, K., O’Rourke, P., Del Mar, C., and Kenardy, J. (2005). Psychological interventions for overweight or obesity. Cochrane Database Syst. Rev. CD003818. doi: 10.1002/14651858.CD003818.pub2

Shaw, R. J., Bosworth, H. B., Silva, S. S., Lipkus, I. M., Davis, L. L., Sha, R. S., et al. (2013). Mobile health messages help sustain recent weight loss. Am. J. Med. 126, 1002-1009. doi: 10.1016/j.amjmed.2013.07.001

Simpson, S. G., and Slowey, L. (2011). Video therapy for atypical eating disorder and obesity: a case study. Clin. Pract. Epidemiol. Ment. Health 7, 38-43. doi: 10.2174/1745017901107010038

Sonneville, K. R., La Pelle, N., Taveras, E. M., Gillman, M. W., and Prosser, L. A. (2009). Economic and other barriers to adopting recommendations to prevent childhood obesity: results of a focus group study with parents. BMC Pediatr. 9:81. doi: 10.1186/1471-2431-9-81

Swencionis, C., and Rendell, S. L. (2012). The psychology of obesity. Abdom. Imaging 37, 733-737. doi: 10.1007/s00261-012-9863-9

Tufano, J. T., and Karras, B. T. (2005). Mobile eHealth interventions for obesity: a timely opportunity to leverage convergence trends. J. Med. Internet Res. 7, e58. doi: 10.2196/jmir.7.5.e58

Turner-McGrievy, G. M., Beets, M. W., Moore, J. B., Kaczynski, A. T., BarrAnderson, D. J., and Tate, D. F. (2013). Comparison of traditional versus mobile app self-monitoring of physical activity and dietary intake among overweight adults participating in an mHealth weight loss program. J. Am. Med. Inform. Assoc. 20, 513-518. doi: 10.1136/amiajnl-2012-001510

Wadden, T. A., Brownell, K. D., and Foster, G. D. (2002). Obesity: responding to the global epidemic. J. Consult. Clin. Psychol. 70, 510-525. doi: 10.1037/0022006X.70.3.510

Waller, G., Stringer, H., and Meyer, C. (2011). What cognitive behavioral techniques do therapists report using when delivering cognitive behavioral therapy for the eating disorders? J. Consult. Clin. Psychol. 80, 171-175. doi: 10.1037/a0026559

Whitfield, M., Bhanbhro, S., Green, G., Lewis, K., Hindle, L., and Levy, C. (2015). Developing a framework for estimating the potential impact of obesity interventions in a European city. Health Promot. Int. doi: 10.1093/heapro/dav019 [Epub ahead of print]

Whitlock, G., Lewington, S., Sherliker, P., Clarke, R., Emberson, J., Halsey, J., et al. (2009). Body-mass index and cause-specific mortality in 900000 adults: collaborative analyses of 57 prospective studies. Lancet 373, 1083-1096. doi: 10.1016/S0140-6736(09)60318-4

Whittaker, R. (2012). Issues in mHealth: findings from key informant interviews. J. Med. Internet Res. 14, e129. doi: 10.2196/jmir.1989

Wilson, G. T., and Schlam, T. R. (2004). The transtheoretical model and motivational interviewing in the treatment of eating and weight disorders. Clin. Psychol. Rev. 24, 361-378. doi: 10.1016/j.cpr.2004.03.003

Wing, R. R. (2002). "Behavioral weight control”, in Handbook of Obesity Treatment, eds W. Ta and S. Aj (New York: Guilford Press), 301-316.

Zimmet, P. Z., Magliano, D. J., Herman, W. H., and Shaw, J. E. (2014). Diabetes: a 21st century challenge. Lancet Diabetes Endocrinol. 2, 56-64. doi: $10.1016 /$ S2213-8587(13)70112-8

Conflict of Interest Statement: The authors declare that the research was conducted in the absence of any commercial or financial relationships that could be construed as a potential conflict of interest.

Copyright (C) 2015 Castelnuovo, Pietrabissa, Manzoni, Corti, Ceccarini, Borrello, Giusti, Novelli, Cattivelli, Middleton, Simpson and Molinari. This is an open-access article distributed under the terms of the Creative Commons Attribution License (CC $B Y)$. The use, distribution or reproduction in other forums is permitted, provided the original author(s) or licensor are credited and that the original publication in this journal is cited, in accordance with accepted academic practice. No use, distribution or reproduction is permitted which does not comply with these terms. 